\title{
Wave Propagation Modeling and Amplitude-Variation-with-Offset Response in a Fractured Coalbed
}

\author{
Qing-Xi LIN ${ }^{1}$, Su-Ping PENG ${ }^{1}$, Wen-Feng DU ${ }^{1}$, \\ Su-Zhen $\mathrm{SHI}^{1}$, and Jing-Wei GOU ${ }^{2}$ \\ ${ }^{1}$ State Key Laboratory of Coal Resources and Mine Safety, China University \\ of Mining \& Technology (Beijing), Beijing, China; e-mail: psp@cumtb.edu.cn \\ ${ }^{2}$ Geophysical Prospecting Institute, \\ China National Administration of Coal Geology, Zhuozhou, China
}

\begin{abstract}
Coalbed methane can be detected employing the amplitudevariation-with-offset technique. However, there are two issues in applying this technique to a coalbed: strong azimuthal anisotropy resulting from high-density fractures, and the seismic response being composed of many or several individual reflections within the coalbed. To overcome these difficulties, we present an exact solution for reflections in extensive dilatancy anisotropy media. First, we build a three-layer model and simulate the wave propagation in this model. Then we derive an exact $P$ - and converted $S$-wave reflection coefficient equation based on boundary conditions. Finally, substituting given model parameters into the exact equation, we obtain the variation in the reflection coefficient with incidence angle. The results show that the fracture factors, wavelet frequency and thickness of the coalbed have different effects on the reflection coefficient. Furthermore, we create a synthetic seismogram by forward calculation, and the result fits well with results of the exact equation.
\end{abstract}

Key words: extensive dilatancy anisotropy, amplitude-variation-withoffset response, wave propagation for wave field modeling, reflection coefficient. 


\section{INTRODUCTION}

In recent decades, coalbed methane (CBM) has become one of the most important future fuel resources in many countries. It has been claimed that the worldwide resources of methane trapped in coal are greater than the total reserves of all known conventional gas fields (Bachu and Bell 2001). Because of its extensive presence in coalbeds, not only can CBM be explored as a clean energy in industrial production, but it is also a hazard in traditional mining. Coal and methane outbursts, which threaten the lives of coal miners, may occur when a coal mining operation breaks into CBM that has accumulated in natural or manmade voids (Hu and McMechan 2007). Therefore, the detection of CBM plays an important role in exploring fuel resources and ensuring the safety of coal miners.

As a direct hydrocarbon-detection technique, the amplitude variation with offset (AVO) technique has been used in the oil and gas industry for more than 20 years. With seismic prestack data, the technique can be employed to predict reservoir properties and the fluid content. The theoretical basis of the AVO technique is the Zoeppritz equation, which was first proposed by Zoeppritz (1919). Zoeppritz calculated the reflection and transmission coefficients of plane waves generated by an incidence wave at a flat interface separating two homogeneous half-spaces. Because the complexity of the problem obscures any physical insight into the AVO signature, simplified or approximate equations have been discussed in the literature (e.g., Aki and Richards 1980, Bortfeld 1961, Shuey 1985). The equations describe the physical phenomenon that provides a possibility of direct hydrocarbon detection. Ostrander (1984) first introduced a practical application of the AVO effect, showing that the presence of gas causes amplitudes to increase with offset. Rutherford and Williams (1989) classified the AVO anomalies relating various AVO phenomena to their geologic and geophysical origins and established a consistent and theoretical AVO-anomaly interpretation system. Castagna et al. (1998) discussed the Rutherford and Williams' classification and proposed a fourth class of sand (class IV). Furthermore, many researchers have studied the AVO research of anisotropic media and obtained a lot of useful conclusions on the detection of cracks and fluid (e.g., Wright 1987, Chen 1995, Bakulin et al. 2000a,b). And now, the AVO technique has drawn substantial attention within the oil industry, eventually becoming a commercial tool.

Although the AVO technique has matured sufficiently to be used in finding oil and gas, it cannot be used directly in CBM detection for three main reasons. First, CBM is a form of natural gas that commonly exists in coalbeds. Unlike traditional natural gas that accumulates in a free state, CBM is stored in deeply buried coal seams by a process called "adsorption" 
in a near-liquid state and lines the inside of pores within the coal (called the matrix). Coal is a porous medium with a dual-pore system comprising microcracks and cleats and micropores. Furthermore, the high-density vertical cracks and fractures contained in a coalbed result in strong seismic anisotropy. Shuck et al. (1996) and Ramos and Davis (1997) studied the multicomponent characterization of a CBM reservoir and demonstrated moderate to strong anisotropy of $30-50 \%$, which is higher than the anisotropy of other sedimentary rocks $(10-20 \%)$. As the theoretical basis of AVO technology, Zoeppritz equation is mainly applied to isotropy and weak anisotropy. In strongly anisotropic environments, where the anisotropic properties are possibly induced by aligned fractures, fillings or other factors, conventional AVO analysis could be distorted significantly and inaccurate. Second, the coalbed is considered to be a thin bed. The seismic response recorded at the surface is composed of many or several individual reflections, including reflections from the top and bottom of the coal seam, converted wave reflections, and multiple reflections (Gochioco 1991, Meissner and Meixner 1969). Third, in the coal industry, the seismic data acquisition requirements (such as sampling, offsets, noise types, etc.) are very different from oil detection. In addition, coal seams can produce distinct reflections because of the exceptionally large impedance contrast with respect to roof and floor rocks. Therefore, AVO analysis is not suitable for CBM detection. We need build a new model suitable for a coalbed instead of applying the conventional AVO to CBM detection directly.

A few researchers have transferred AVO technique to CBM exploration. Ramos and Davis (1997) characterized fractured reservoirs and applied the AVO technique to CBM exploration of the Cedar Hill Field. Peng et al. (2006) compared the strengths and weaknesses of the AVO technique for $\mathrm{CBM}$ reservoirs with those of the AVO technique for gas sand reservoirs and detected rich methane within coal seams. However, the reflection coefficients of the coalbed that are more important for describing AVO responses have not been calculated using a set of exact formulae. Wave propagation should be analyzed to investigate the characteristics of elastic wave propagation in a fractured coalbed. A formula can then be derived to calculate the reflection coefficients.

In this study, we analyzed the fractured coalbed with strong anisotropy. We consider the coalbed to be an extensive dilatancy anisotropic (EDA) medium, which is typically produced by a system of vertical cracks. We used Cheng's (1993) model and the Kelvin-Christoffel equations to estimate the elastic parameters of the EDA medium and compute the wave velocity, respectively. A three-layer medium model (the EDA medium spread between two isotropic layers) was then designed with the cracks along the $90^{\circ}$ azimuth. The elastic wave propagation was simulated at azimuthal angles of $0^{\circ}$ 
and $90^{\circ}$. We derived an exact formula from the Zoeppritz equation to calculate the reflection coefficients with high precision. When the physical parameters of the coalbed are estimated for the model, the change in $P$ - and converted $S$-wave reflection coefficients with different fracture factors can be analyzed.

\section{THEORETICAL ASPECTS}

\subsection{Anisotropy of the coalbed}

It is widely known that anisotropy pervades most rocks in the Earth's crust. Coal is an unusual reservoir rock that has an impermeable and isotropic matrix together with a fabric of natural fractures known as cleats or cracks that most likely causes the observed anisotropy. It is important to model the coalbed with layers of elastic anisotropy when we describe the wave propagation through a fractured coalbed.

Crampin (1989) classified anisotropy into 10 groups according to the symmetry of the medium produced by the wave driving force. One class of anisotropy, EDA, was firstly proposed by Crampin (1984). He pointed out that stress-induced cracks would be open in the direction of maximum stress and closed in the direction of minimum stress. Stress-aligned near-parallel near-vertical cracks are thus formed. EDA is used frequently to describe a system of parallel and vertically aligned penny-shaped cracks embedded in an isotropic matrix, and has hexagonal symmetry with a horizontal axis of symmetry, parallel to the minimum horizontal compressional stress.

Generally, coalbeds are equivalent to EDA media because the aligned cracks contained by coalbeds are always distributed parallel to each other. A sketch of an EDA model is shown in Fig. 1. Research on the layout and anisotropy of EDA media helps clarify the factors affecting CBM.

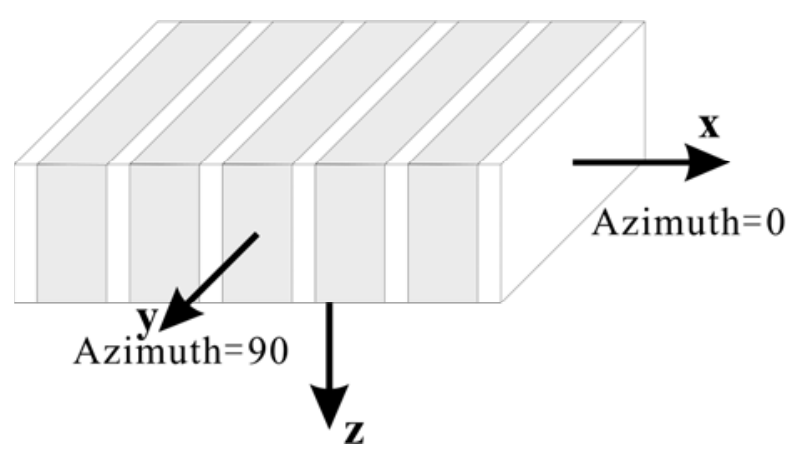

Fig. 1. Sketch of an EDA model. 


\subsection{Computation of the stiffness tensor}

The effective anisotropic stiffness tensor is a mathematical expression of anisotropy and is important in describing fractured rocks. Coal has a much higher fracture density than other sedimentary rocks. High fracture density leads to stronger anisotropy. In consideration of the strong anisotropy, it is important to choose a parameterization model to compute the stiffness tensor of anisotropic coal. There are two models commonly used to describe the fracture density of coal: the Hudson (1981) and the Cheng (1993) models.

The widely accepted model for fracture-induced anisotropy is that of Hudson $(1980,1981)$. This model mainly uses Eshelby's (1957) technique for calculating the elastic constants of cracked solids and the effects on seismic waves of scattering at penny-shaped ellipsoidal fractures or inclusions.

Hudson's theory computes the stiffness tensor from the background rock moduli, crack density, aspect ratio, and physical parameters of material inside cracks. The crack density is defined as:

$$
\varepsilon=N a^{3} / V,
$$

where $N$ is the number of cracks of radius $a$ in a volume $V$. The Hudson second-order expression for the stiffness matrix $\mathbf{C}^{*}$ is:

$$
\mathbf{C}^{*}=\mathbf{C}^{0}+\mathbf{C}^{1}+\mathbf{C}^{2},
$$

where $\mathbf{C}^{0}$ is the stiffness tensor of the host rock (isotropic background rock). The first-order term $\mathbf{C}^{1}$ accounts for single scattering and the second-order term $\mathbf{C}^{2}$ gives the crack-crack interactions. Both $\mathbf{C}^{1}$ and $\mathbf{C}^{2}$ are perturbations of the isotropic stiffness tensor $\mathbf{C}^{0}$ of the uncracked solid.

Hudson's second-order formulation has been used by Crampin (1984) and other researchers to model anisotropy of seismic waves propagating through EDA cracks. However, it has been found that Hudson's approximation is inaccurate for high crack densities. This is because the second-order terms can dominate the first-order terms at higher crack densities and the effective moduli increases with increasing crack density, as shown in Fig. 2a. Generally, it is assumed that this model works when the crack density is less than 0.1 .

To overcome the existing limits, Cheng's (1993) idea was to extend Hudson's theory to high crack density and reformulate the expansion as a Padé series. The problem of divergence at higher crack densities can be avoided using this Padé-approximation-based expansion. The improved formulation for calculating the stiffness tensor is expressed as:

$$
\mathbf{C}^{*}=\mathbf{C}^{0}(1-\mathbf{A} \varepsilon) /(1+\mathbf{B} \varepsilon),
$$


(a) Dry fractures

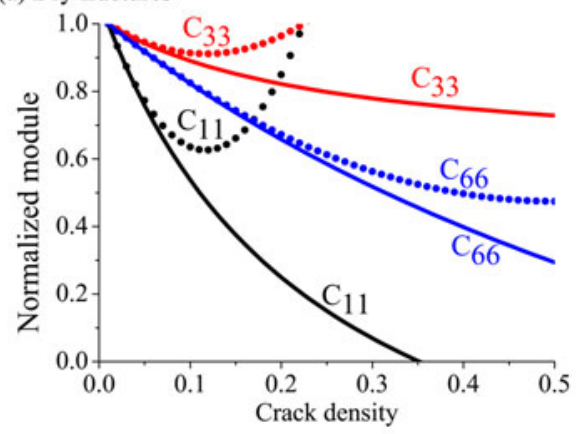

(c) Water-saturated fractures

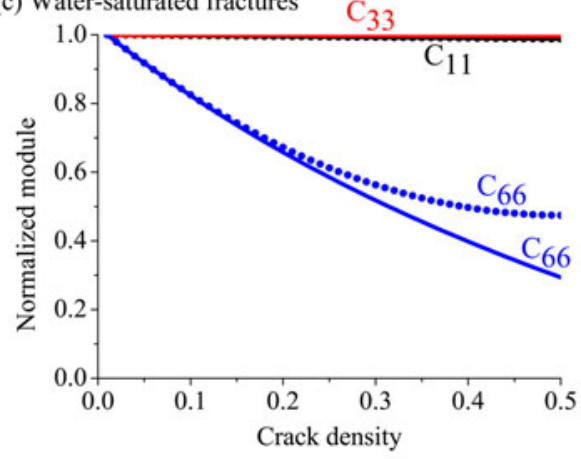

(b) Gas-saturated fractures

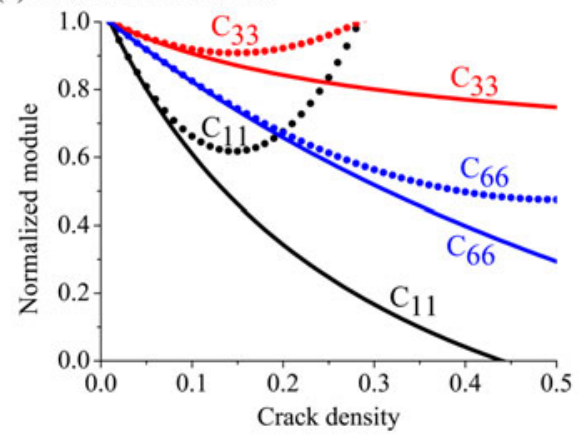

Fig. 2. Comparison of the normalized elastic modulus calculated with Hudson's (dashed line) and Cheng's (solid line) models with different fracture densities for dry fractures (a), gassaturated fractures (b), and watersaturated fractures (c).

where $\varepsilon$ is the crack density. Padé coefficient matrixes $\mathbf{A}$ and $\mathbf{B}$ are defined as:

$$
\mathbf{A}=-\mathbf{C}^{1} /\left(\mathbf{C}^{0} \varepsilon\right)-\mathbf{B}, \quad \mathbf{B}=-\mathbf{C}^{2} /\left(\mathbf{C}^{1} \varepsilon\right) .
$$

We computed the elastic constants for the anisotropic layers using Hudson's and Cheng's theories for the properties of the fractured layer. Figure 2 shows the elastic constants $C_{11}, C_{33}$, and $C_{66}$ against crack density of the coalbed with dry, gas-saturated, and water-saturated cracks. The dashed lines and solid lines were calculated by Hudson's second-order formulation and Cheng's expansion, respectively. The background $P$ - and $S$-wave velocities of the coalbed were, respectively, $v_{p 0}=2200 \mathrm{~m} / \mathrm{s}$ and $v_{s 0}=1100 \mathrm{~m} / \mathrm{s}$, and the density was $\rho_{0}=1390 \mathrm{~kg} / \mathrm{m}^{3}$. The average aspect ratio of cracks was 0.002 . The bulk moduli of the gas and water that filled the fractures were 2 and $2250 \mathrm{MPa}$, respectively. The azimuth of the cracks is $90^{\circ}$ (shown in Fig. 1). From Fig. 2, we see that Hudson's expansion becomes physical for water-saturated cracks but unphysical for dry and gas-saturated cracks when the crack density is beyond 0.1. Meanwhile, Cheng's formulation agrees well for coal over a large range of crack densities of up to 0.35 . 


\subsection{Wave propagation in EDA media}

In the isotropic media, velocity of $P$ and $S$ waves do not vary with direction of propagation. The polarization directions are mutually orthogonal to each other. A $P$ wave is polarized along its propagation direction. The polarization direction of an $S$ wave is orthogonal to the propagation direction.

In EDA media, wave propagation adds some new challenges. First, shear wave splitting can occur when a polarized shear wave enters an EDA medium and splits into two polarized shear waves: $S V$ and $S H$. One of the shear waves is faster than the other owing to the cracks in the medium. Second, a qualifier quasi is always written in front of " $P$ wave" and " $S$ wave" because the longitudinal wave or shear wave is not polarized normal to either the slowness or propagation directions for EDA media. Third, the wave velocity is dependent on propagation and polarization directions.

According to the above, the derivation of wave velocities and that of polarization vectors are two necessary elements in studying wave propagation in EDA media. In this paper, we calculated the seismic wave velocities and associated polarization vectors from the solution of Kelvin-Christoffel equations for an arbitrary propagation direction (Appendix A). As described in Appendix A, crack-induced anisotropy has an important effect on wave velocity.

\section{EXACT SOLUTION FOR REFLECTIONS IN EDA MEDIA}

To study the AVO response in a fractured coalbed, we need to establish a geological model and present solutions to the reflection problem. A coalbed is well known to be a layer of sedimentary rock buried between overlaying and underlying strata. The AVO response of the coalbed, which is always considered as a thin bed, is composed of many reflections. Wave propagation in layered isotropic media has been studied by many researchers (e.g., Du 1996), and an exact formula has been derived for calculating reflection coefficients. However, strong anisotropy is presented by the quite large crack density of the coalbed and the isotropic model is unable to provide accurate results. Therefore, we have to build an anisotropic model.

A three-layer model was established and is shown in Fig. 3b. The top and bottom layers are isotropic, while the middle layer is an EDA medium. To simplify the calculation, wave propagation is only simulated at azimuthal angles of $0^{\circ}$ and $90^{\circ}$. In Figure 3b, the symmetry axis of cracks is parallel to the $x$ direction. The $[x, z]$-plane is referred to as the "symmetry-axis plane", and the $[y, z]$-plane where waves do not experience any anisotropy is the socalled "isotropy plane" (Rüger 1997). We define that a plane wave propagates in the $[x, z]$-plane and $[y, z]$-plane when the azimuthal angles are $0^{\circ}$ and $90^{\circ}$, respectively. 


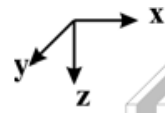

(a)

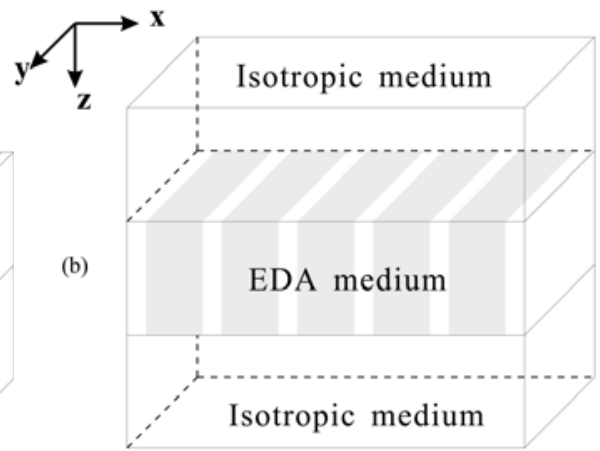

Fig. 3. Geological models of the coalbed: (a) a two-layer model with two EDA media, and (b) a three-layer model with two isotropic media and an EDA medium.

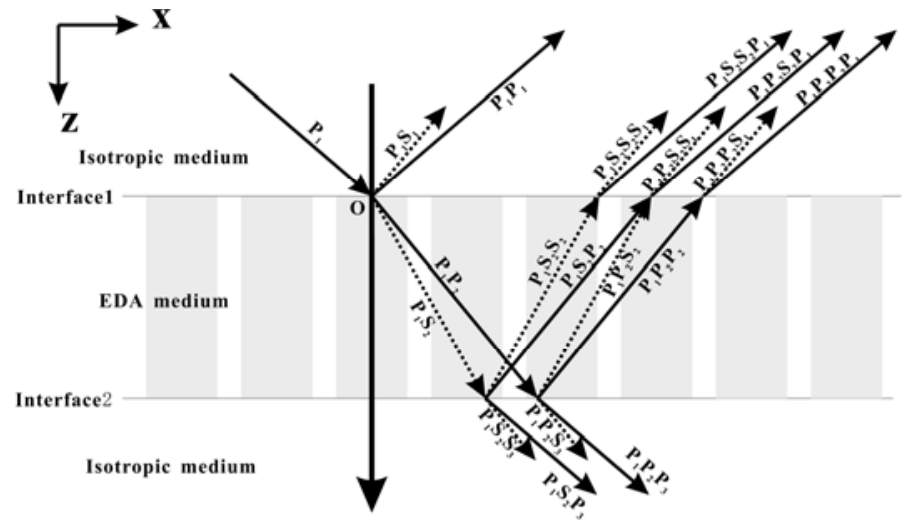

Fig. 4. Wave paths of plane elastic waves propagating in the fractured coalbed.

In Appendix B, a different model (upper and lower layers are both an anisotropic medium) is established (Fig. 3a). One can illustrate the characteristics of the wave motion and obtain the reflection coefficients at the interface. Then, as an extension of the above studies, an exact solution for reflections was obtained for the three-layer model (Fig. 3b).

First, we will study the wave propagation in the three-layer model for an azimuthal angle of $0^{\circ}$. When a plane $P$ wave is incident at interface 1 (shown in Fig. 4), it is reflected on the upper and lower boundaries of the coalbed. The observed reflection is a composite of the reflections from the top and bottom of the coalbed and all multiple and converted waves reflections within the coalbed. However, in the highly attenuated layer the influence of the attenuation is the highest for the multiple reflections. For this reason, we de- 
cided to neglect their presence in our study. Figure 4 shows all the reflections and transmissions when waves propagate into the fractured coalbed.

In Figure 4, there are 10 reflected waves in the upper isotropic layer when waves pass through the fractured coalbed: reflected $P$ waves $P_{1} P_{1}$, $P_{1} S_{2} S_{2} P_{1}, P_{1} S_{2} P_{2} P_{1}, P_{1} P_{2} S_{2} P_{1}$, and $P_{1} P_{2} P_{2} P_{1}$, and converted $S$ waves $P_{1} S_{1}$, $P_{1} S_{2} S_{2} S_{1}, P_{1} S_{2} P_{2} S_{1}, P_{1} P_{2} S_{2} S_{1}$, and $P_{1} P_{2} P_{2} S_{1}$. Note that waves $P_{1} S_{2} P_{2} P_{1}$ and $P_{1} S_{2} P_{2} S_{1}$ are not shown in Fig. 4, as their propagation paths coincide with those of $P_{1} P_{2} S_{2} P_{1}$ and $P_{1} P_{2} S_{2} S_{1}$.

To explain the wave paths in Fig. 4, we apply a finite difference method to simulate the elastic wave propagating in the three-layer model. This method has fourth-order accuracy in space and second-order accuracy in time. In the numerical example, the thickness of each layer is $100 \mathrm{~m}$. The $P$-wave velocities, $S$-wave velocities, and densities in the top and bottom layers are $v_{p 1}=v_{p 3}=3710 \mathrm{~m} / \mathrm{s}, \quad v_{s 1}=v_{s 3}=1990 \mathrm{~m} / \mathrm{s}, \quad$ and $\quad \rho_{1}=\rho_{3}=$ $2.6 \mathrm{~g} / \mathrm{cm}^{3}$, respectively, whereas in the middle layer, the background parameters are $v_{p 2}=2200 \mathrm{~m} / \mathrm{s}, \quad v_{s 2}=1100 \mathrm{~m} / \mathrm{s}$, and $\rho_{2}=1.39 \mathrm{~g} / \mathrm{cm}^{3}$. The crack density is 0.3 and the aspect ratio of the dry crack is 0.002 . The azimuth of the cracks is $90^{\circ}$. The grid intervals are $\Delta x=\Delta z=1 \mathrm{~m}$. The time sampling interval is $\Delta t=0.1 \mathrm{~ms}$. The $P$-wave source with a frequency of $150 \mathrm{~Hz}$ is located at the center of the model. In this experiment, we use the classical perfectly matched layer (Komatitsch and Tromp 2003) as the absorbing boundary condition to eliminate reflections at the artificial boundaries. Figure 5 shows snapshots of elastic wavefields at different times. Some wave types shown in Fig. 4 are observed in these snapshots. Note that the snapshot time is stopped at $120 \mathrm{~ms}$, because the conventional finite difference grids may become unstable after seismic waves run for long distances and a long time.

Next, the exact formula for reflection coefficients needs to be derived. As shown in Fig. 4, the propagation directions of all $P$ waves are parallel to each other in the upper isotropic layer. Thus, these $P$ waves have the same polarization, horizontal slowness, and vertical slowness, similar to the case for the $S$ waves propagating in the upper isotropic layer. The displacement vector of any of the mentioned waves at interface 1 can be expressed as:

$$
\begin{gathered}
\mathrm{u}_{p m}=\left(f_{p}, h_{p}\right) A_{p m} \exp \left[i \omega\left(p x+r_{p} z-t\right)\right] \exp \left(i \phi_{p m}\right), \quad m=1,2, \ldots, 5, \\
\mathrm{u}_{s n}=\left(f_{s}, h_{s}\right) A_{s n} \exp \left[i \omega\left(p x+r_{s} z-t\right)\right] \exp \left(i \phi_{s n}\right), \quad n=1,2, \ldots, 5,
\end{gathered}
$$

where the subscript $m=1,2, \ldots, 5$ corresponds to the $P$ waves $P_{1} P_{1}$, $P_{1} S_{2} S_{2} P_{1}, P_{1} S_{2} P_{2} P_{1}, P_{1} P_{2} S_{2} P_{1}$, and $P_{1} P_{2} P_{2} P_{1}, n=1,2, \ldots, 5$ corresponds to the converted $S$ waves $P_{1} S_{1}, P_{1} S_{2} S_{2} S_{1}, P_{1} S_{2} P_{2} S_{1}, P_{1} P_{2} S_{2} S_{1}$, and $P_{1} P_{2} P_{2} S_{1}$, $\left(f_{p}, h_{p}\right)$ and $\left(f_{s}, h_{s}\right)$ are unit polarization vectors of $P$ and $S$ waves, $p$ is hori- 

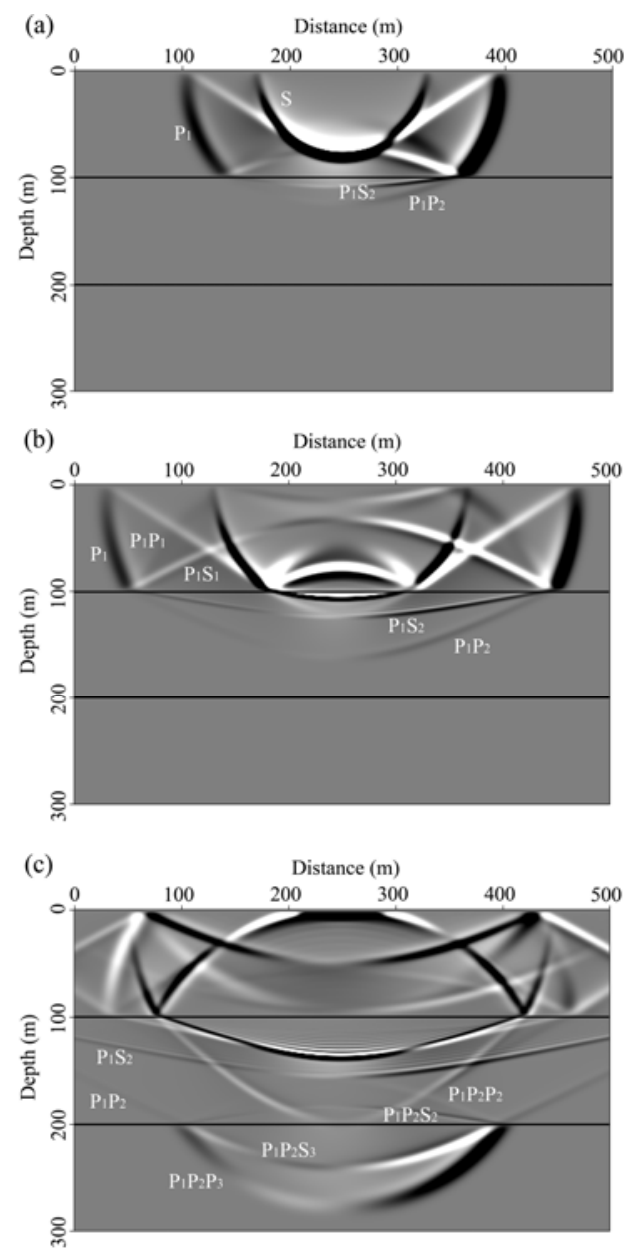

Fig. 5. Snapshots of the $X$-component at different times in the dry fractured coalbed model: (a) $X$-component snapshot at $t=$ $60 \mathrm{~ms}$, showing the incident $P$ and $S$ waves, and the transmitting $P$ wave and converted $S$ wave from the $P$ wave on the coalbed roof; (b) $X$-component snapshot at $t=80 \mathrm{~ms}$, showing the reflected $P$ wave and converted $S$ wave on the coalbed roof; (c) $X$-component snapshot at $t=120 \mathrm{~ms}$, showing the reflected $P$ wave and converted $S$ wave from $P_{1} P_{2}$ on the coalbed floor, as well as transmitted waves.

zontal slowness, $r_{p}$ and $r_{s}$ are values of the vertical slowness of $P$ and $S$ waves, and $\varphi_{p m}$ and $\varphi_{s n}$ are the initial wavefront phases of $P$ and $S$ waves.

Assuming that the phase of the $P_{1}$ wave on arrival at interface 1 is zero, we obtain:

$$
\phi_{p 1}=\phi_{s 1}=0 \text {. }
$$

The initial wavefront phases of other waves are constrained by the travel time delay. As an example, the initial phase of $P_{1} P_{2} P_{2} P_{1}$ (i.e., $\left.\varphi_{p 5}\right)$ can be calculated in the next step.

Figure 6 shows the ray paths of $P$-waves $P_{1}, P_{1} P_{2}, P_{1} P_{2} P_{2}$, and $P_{1} P_{2} P_{2} P_{1}$. The incidence angle of wave $P_{1}$ is $\theta_{1}$ while the transmission angle is $\theta_{2}$. 


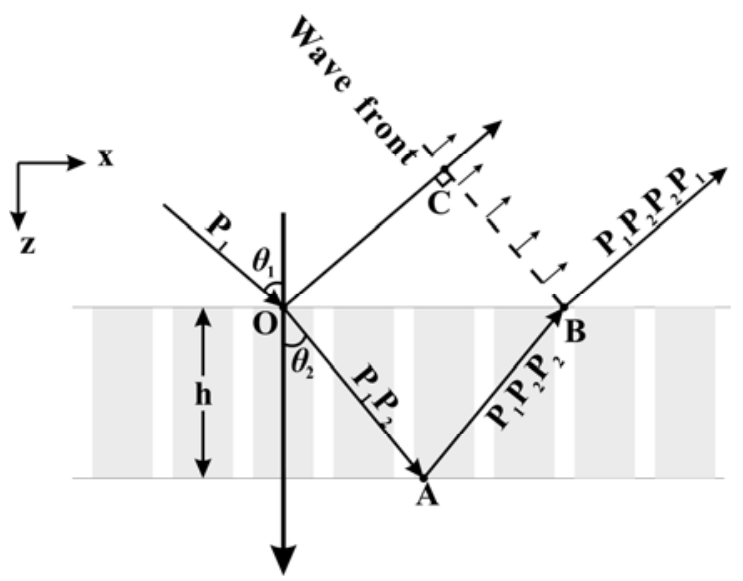

Fig. 6. Ray paths of $P$ waves.

The thickness of the coalbed is $h$. The delay time $\Delta t_{O C}$ can be calculated according to:

$$
\Delta t_{O C}=\overline{O C} / v_{1}=\left(\overline{O B} \sin \theta_{1}\right) / v_{1}=\left(2 h \sin ^{2} \theta_{2}\right) /\left(v_{2} \cos \theta_{2}\right),
$$

where $v_{1}$ is the velocity of the $P$ wave propagating in the upper isotropic layer, and $v_{2}$ is the velocity of $P$ waves $P_{1} P_{2}$ and $P_{1} P_{2} P_{2} . \Delta t_{O A B}$ is calculated as:

$$
\Delta t_{O A B}=\Delta t_{O A}+\Delta t_{A B}=2 \overline{O A} / v_{2}=2 h /\left(v_{2} \cos \theta_{2}\right) .
$$

The travel time delay between reflected $P$ waves is given by:

$$
\Delta t=\Delta t_{O A B}-\Delta t_{O C}=2 h /\left(v_{2} \cos \theta_{2}\right)-\left(2 h \sin ^{2} \theta_{2}\right) /\left(v_{2} \cos \theta_{2}\right)=\left(2 h \cos \theta_{2}\right) / v_{2} \text {. }
$$

The phase difference (i.e., the initial phase of $P_{1} P_{2} P_{2} P_{1}$ ) is given by:

$$
\phi_{p 5}=\omega \Delta t=\omega\left(2 h \cos \theta_{2}\right) / v_{2} .
$$

The initial phase of other waves can be calculated in the same way.

The amplitude of the reflection is governed by the transmission and reflection coefficients of the top and bottom interfaces. Equations 5 and 6 can be written as:

$$
\begin{gathered}
\mathrm{u}_{p m}=\left(f_{p}, h_{p}\right) A_{p} R_{p m} \exp \left[i \omega\left(p x+r_{p} z-t\right)\right] \exp \left(i \phi_{p m}\right), \quad m=1,2, \ldots, 5, \\
\mathrm{u}_{\mathrm{s} n}=\left(f_{s}, h_{s}\right) A_{p} R_{s n} \exp \left[i \omega\left(p x+r_{s} z-t\right)\right] \exp \left(i \phi_{s n}\right), \quad n=1,2, \ldots, 5,
\end{gathered}
$$

where $A_{p}$ is the amplitude of incident wave $P_{1}$. When $m=n=1$, we have: 


$$
R_{p 1}=R_{p 1 p 1}, \quad R_{s 1}=R_{p l s 1},
$$

where $R_{p 1 p 1}$ denotes the $P$-wave reflection coefficient of the $P_{1}$ wave at interface $1, R_{p 1 s 1}$ denotes the $S$-wave reflection coefficient of $P_{1}$ at interface 1 . When $m=n=2$, we have:

$$
R_{p 2}=T_{p 1 s 2} R_{p 1 s 2 s 2} T_{p 1 s 2 s 2 p 1}, \quad R_{s 2}=T_{p 1 s 2} R_{p 1 s 2 s 2} T_{p 1 s 2 s 2 s 1},
$$

where $T_{p 1 s 2}$ denotes the $S$-wave transmission coefficient of $P_{1}$ at interface 1, $R_{p 1 s 2 s 2}$ denotes the $S$-wave reflection coefficient of $P_{1} S_{2}$ at interface 2, $T_{p 1 s 2 s 2 p 1}$ denotes the $P$-wave transmission coefficient of $P_{1} S_{2} S_{2}$ at interface 1, and $T_{p 1 s 2 s 2 s 1}$ denotes the $S$-wave transmission coefficient of $P_{1} S_{2} S_{2}$ at interface 1 . These reflection and transmission coefficients can be calculated using Eq. B14 derived in Appendix B.

Similarly, we have:

$$
\begin{array}{ll}
R_{p 3}=T_{p 1 s 2} R_{p 1 s 2 p 2} T_{p 1 s 2 p 2 p 1}, & R_{s 3}=T_{p 1 s 2} R_{p 1 s 2 p 2} T_{p 1 s 2 p 2 s 1}, \\
R_{p 4}=T_{p 1 p 2} R_{p 1 p 2 s 2} T_{p 1 p 2 s 2 p 1}, & R_{s 4}=T_{p 1 p 2} R_{p 1 p 2 s 2} T_{p 1 p 2 s 2 s 1}, \\
R_{p 5}=T_{p 1 p 2} R_{p 1 p 2 p 2} T_{p 1 p 2 p 2 p 1}, & R_{s 5}=T_{p 1 p 2} R_{p 1 p 2 p 2} T_{p 1 p 2 p 2 s 1} .
\end{array}
$$

We assume that the depth of interface 1 is zero. Each wave propagating in the upper isotropic layer, except for incident wave $P 1$, contributes to the total reflected waveform so that the composite reflection of the $P$ wave is given by:

$$
\mathbf{u}_{\mathbf{p} \Sigma}=\sum_{m=1}^{5} \mathbf{u}_{\mathbf{p} m}=\left(f_{p}, h_{p}\right) A_{p} \sum_{m=1}^{5}\left[R_{p m} \exp \left(i \varphi_{p m}\right)\right] \exp [i \omega(p x-t)] .
$$

Using Euler's formula, we can simplify Eq. 19 as:

$$
\mathbf{u}_{\mathbf{p} \Sigma}=\left(f_{p}, h_{p}\right) A_{p} R_{p} \exp \left(i \phi_{p}\right) \exp [i \omega(p x-t)],
$$

where

$$
\begin{gathered}
R_{p}=\sqrt{M_{p}^{2}+N_{p}^{2}}, \quad \phi_{p}=\tan ^{-1} \frac{M_{p}}{N_{p}}, \\
M_{p}=\sum_{m=1}^{5}\left(R_{p m} \cos \varphi_{p m}\right), \quad N_{p}=\sum_{m=1}^{5}\left(R_{p m} \sin \varphi_{p m}\right) .
\end{gathered}
$$

The amplitude and initial phase of composite reflected $P$ waves are $A_{p} R_{p}$ and $\phi_{p}$, respectively. $R_{p}$ is the $P$-wave reflection coefficient.

Similarly, we add the displacements of $S$ waves and obtain:

$$
\mathbf{u}_{\mathrm{s} \Sigma}=\sum_{n=1}^{5} \mathbf{u}_{\mathrm{s} n}=\left(f_{s}, h_{s}\right) A_{p} R_{s} \exp \left(i \phi_{s}\right) \exp [i \omega(p x-t)],
$$


where

$$
\begin{gathered}
R_{s}=\sqrt{M_{s}^{2}+N_{s}^{2}}, \quad \phi_{s}=\tan ^{-1} \frac{M_{s}}{N_{s}}, \\
M_{s}=\sum_{n=1}^{5}\left(R_{s n} \cos \varphi_{s n}\right), \quad N_{s}=\sum_{n=1}^{5}\left(R_{s n} \sin \varphi_{s n}\right) .
\end{gathered}
$$

The amplitude and initial phase of composite reflected $S$ waves are $A_{p} R_{S}$ and $\phi_{s}$, respectively. $R_{s}$ is the converted $S$-wave reflection coefficient.

The same derivation is applied to the solution for reflection coefficients when a wave propagates in the $[y, z]$-plane (azimuth of $90^{\circ}$ ).

The exact solution for reflections has been derived in Appendix B. In the next steps, forward modeling can be employed to compute the reflection coefficients and an accurate AVO response can be obtained.

\section{AVO RESPONSE IN A FRACTURED COALBED}

The AVO response refers to the variation of the seismic wave amplitude or reflection coefficient with offset or incidence angle. The exact formulation has been derived for calculating the reflection coefficient in a three-layer model as discussed above. In this section, we substitute various physical parameters into the equation and analyze the variation in the reflection coefficient with the incidence angle of the $P$ wave. The physical parameters of the three-layer model are listed in Table 1. The values of crack density, crack aspect ratio, bulk modulus of the crack filler, wavelet frequency, and thickness of the coalbed, which are all listed in the table, can be used to analyze the characteristics of the AVO response.

Table 1

\begin{tabular}{|c|c|c|c|c|c|c|c|c|}
\hline \multirow[b]{2}{*}{ Layer } & \multicolumn{3}{|c|}{ Background parameters } & \multirow{2}{*}{$\begin{array}{l}\text { Crack } \\
\text { density }\end{array}$} & \multirow{2}{*}{$\begin{array}{c}\text { Crack } \\
\text { aspect } \\
\text { ratio }\end{array}$} & \multirow{2}{*}{$\begin{array}{l}\text { Crack } \\
\text { filler }\end{array}$} & \multirow{2}{*}{$\begin{array}{c}\text { Wavelet } \\
\text { frequency } \\
{[\mathrm{Hz}]}\end{array}$} & \multirow{2}{*}{$\begin{array}{c}\text { Thick- } \\
\text { ness } \\
{[\mathrm{m}]}\end{array}$} \\
\hline & $\begin{array}{c}v_{p} \\
{[\mathrm{~m} / \mathrm{s}]}\end{array}$ & $\begin{array}{c}v_{s} \\
{[\mathrm{~m} / \mathrm{s}]}\end{array}$ & $\begin{array}{c}\rho \\
{\left[\mathrm{g} / \mathrm{cm}^{3}\right]}\end{array}$ & & & & & \\
\hline $\begin{array}{l}\text { Top } \\
\text { layer }\end{array}$ & 3710 & 1990 & 2.60 & & & & & 100 \\
\hline Coalbed & 2200 & 1100 & 1.39 & $\begin{array}{l}0.15 \\
0.20 \\
0.25 \\
0.30 \\
0.35\end{array}$ & $\begin{array}{l}0.001 \\
0.002 \\
0.003\end{array}$ & $\begin{array}{c}\text { dry } \\
\text { gas } \\
\text { water }\end{array}$ & $\begin{array}{l}40 \\
50 \\
60 \\
70 \\
80\end{array}$ & $\begin{array}{c}3 \\
5 \\
7 \\
9 \\
11\end{array}$ \\
\hline $\begin{array}{c}\text { Bottom } \\
\text { layer }\end{array}$ & 3710 & 1990 & 2.60 & & & & & 100 \\
\hline
\end{tabular}

Physical parameters of the three-layer model

Note: The bulk moduli of gas and water that fill the fractures are 2 and $2250 \mathrm{MPa}$. 

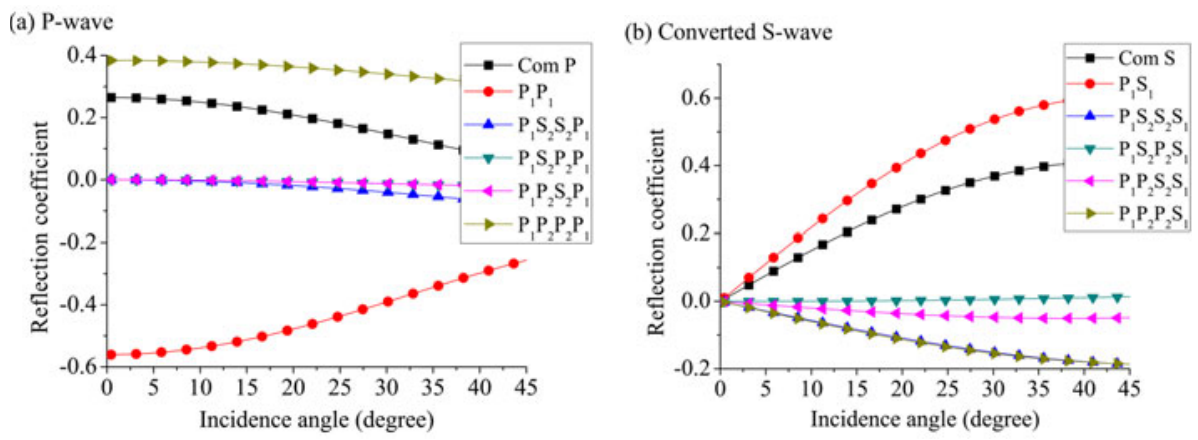

Fig. 7. Effect of a single wave on the reflection response of the composite wave.

We first discuss the characteristics of the variation of reflection coefficients at an azimuthal angle of $0^{\circ}$. From Section 3, we know that the reflection coefficient is a composite of the reflections from the top and bottom of the coalbed in the three-layer model. Thus, we need to analyze the effect of each single wave on the reflection coefficient of the composite wave. Figure 7 shows the $P$-wave and converted $S$-wave reflection coefficients evaluated at interface 1 with gas-saturated fractures. The crack density is set to 0.3 , the average aspect ratio of cracks is 0.002 , the wavelet frequency is $60 \mathrm{~Hz}$, and the thickness of the coalbed is $7 \mathrm{~m}$.

In Figure 7a, the reflection coefficients of $P$ waves $P_{1} P_{1}$ and $P_{1} P_{2} P_{2} P_{1}$ decrease but those of other single waves increase as the incidence angle increases. It is obvious that the $P$ waves $P_{1} P_{1}$ and $P_{1} P_{2} P_{2} P_{1}$ have considerable influence on the reflection coefficient of the composite $P$ wave. Therefore, the reflection coefficient of the composite $P$ wave (shown as "Com $P$ ") decreases with an increasing incidence angle. In Figure $7 \mathrm{~b}$, the $S$-wave reflection coefficients of all converted $S$ waves appear to have an increasing trend. Additionally, the converted wave $P_{1} S_{1}$ has notably affects in the reflection coefficient of the composite $S$ wave (shown as "Com $S$ "). From the discussion above, we conclude that the reflection from the top of the coalbed strongly affects the reflection coefficients of composite waves.

Next, we consider factors, such as the crack density, crack aspect ratio (thickness over length, namely, minor/major axis of ellipsoid), bulk modulus of the crack filler, wavelet frequency and thickness of the coalbed that affect the AVO response. First, we vary the crack density from 0.15 to 0.35 at intervals of 0.05 . The average aspect ratio of cracks is 0.002 , the crack filler is gas, the wavelet frequency is $60 \mathrm{~Hz}$, and the thickness of the coalbed is $7 \mathrm{~m}$. Figure 8 shows the effect of crack density on the reflection coefficient.

In Figure 8a, both the intercept and slope of the $P$-wave reflection coefficient curves increase with increasing crack density. The reflection coeffi- 


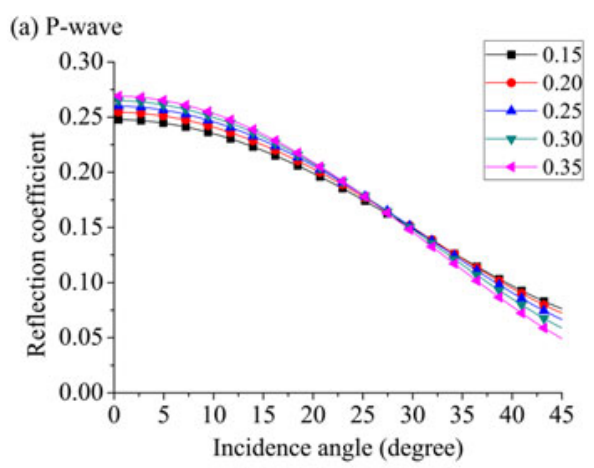

(b) Converted S-wave

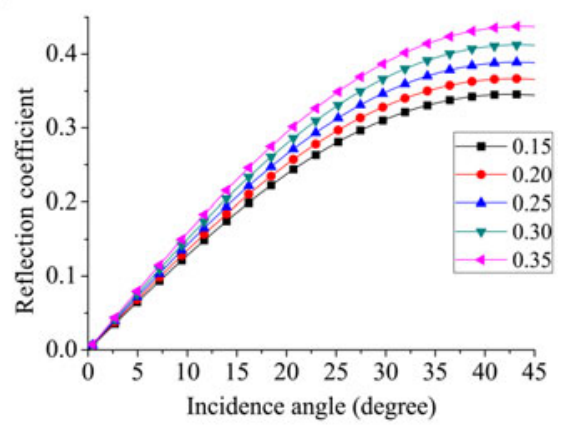

Fig. 8. Effect of crack density on the wave reflection coefficient.

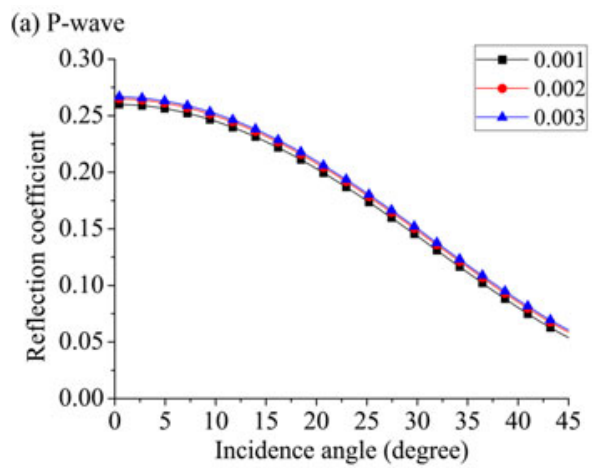

(b) Converted S-wave

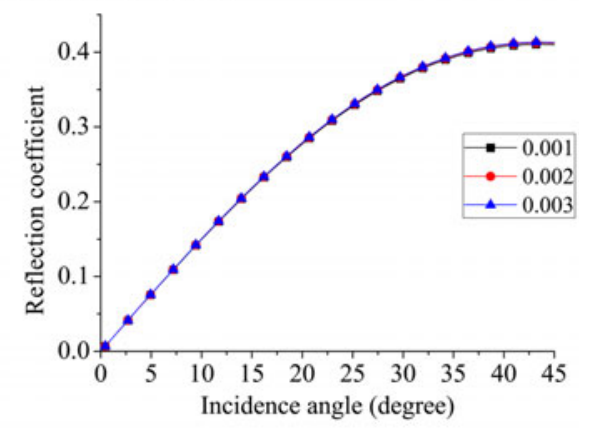

Fig. 9. Effect of the crack aspect ratio on the wave reflection coefficient.

cient curves resulting from different crack densities tend to equal each other when the incidence angle is about $27^{\circ}$. When the incidence angle is larger than $27^{\circ}$, the reflection coefficient decreases with increasing crack density. Figure $8 \mathrm{~b}$ shows that the reflection coefficient of the converted $S$ wave increases with increasing crack density.

The average aspect ratio of cracks affects the stiffness tensor of the coalbed, as well as the reflection coefficient. Figure 9 shows the effect of the crack aspect ratio, which varies from 0.001 to 0.003 in intervals of 0.05 , on the reflection coefficient. The crack density is 0.3 and the other parameters are the same as above.

In general, the crack aspect ratio has little effect on the reflection coefficient. For the $P$ wave, the intercept of the reflection coefficient curve increases with increasing crack aspect ratio, but the slope does not substantially change. For the converted $S$ wave, there is a fractional change in the reflection coefficient. 


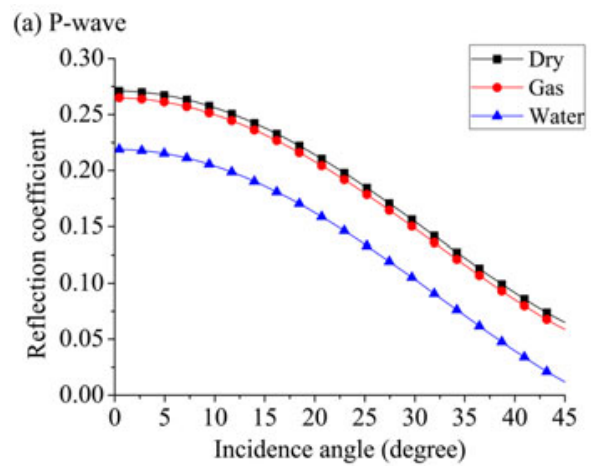

(b) Converted S-wave

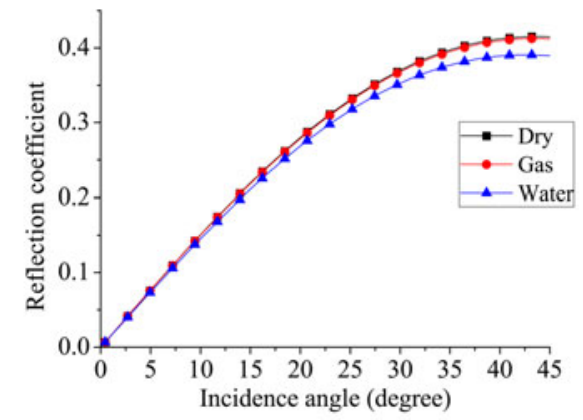

Fig. 10. Effect of the crack filler on the wave reflection coefficient.

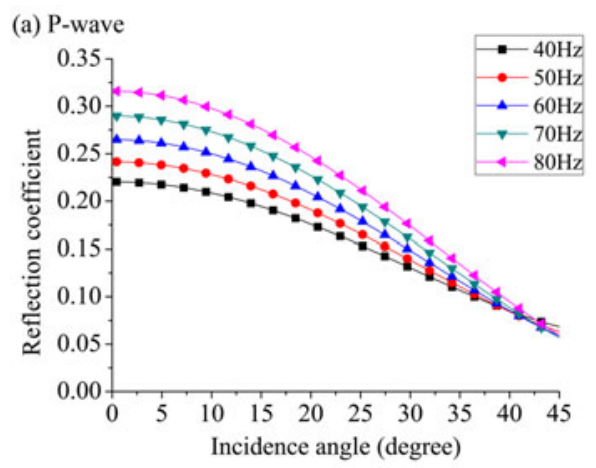

(b) Converted S-wave

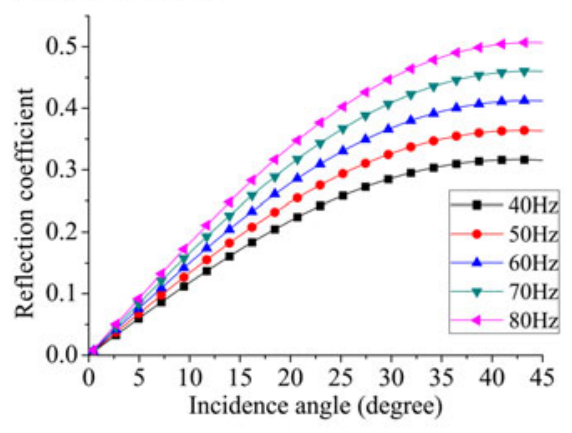

Fig. 11. Effect of wavelet frequency on the wave reflection coefficient.

In the next section we will discuss the effect on the reflection coefficient induced by the dry, gas or water saturated cracks. Figure 10 shows the incidence angle against the wave reflection coefficient for dry, gas-saturated, and water-saturated cracks. The bulk moduli were listed in Table 1. The crack density is 0.3 , the average aspect ratio of cracks is 0.002 , the wavelet frequency is $60 \mathrm{~Hz}$, and the thickness of the coalbed is $7 \mathrm{~m}$.

In Figure 10a, the reflection of the $P$ wave is smaller when the crack is filled with gas or water than dry cracks. This is especially visible in the case of the water-saturated cracks. The same change is observed for the converted $S$ wave in Fig. 10b. The wave reflection coefficient is little affected by the gas saturated cracks but strongly affected by the water saturated cracks.

Next, we will study the change of the wavelet frequency from 40 to $80 \mathrm{~Hz}$ at $10-\mathrm{Hz}$ intervals. The crack filler is gas and the other parameters are the same as previously. The wave reflection coefficient changes are shown in Fig. 11. 

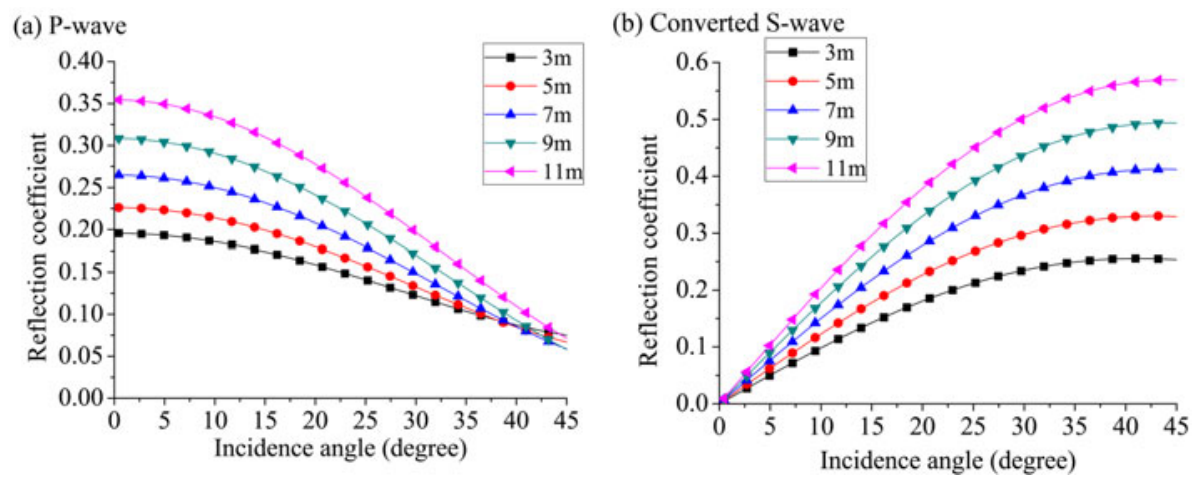

Fig. 12. Effect of the coalbed thickness on the wave reflection coefficient.

For the $P$ wave, the intercept and slope of the reflection coefficient curves increase with increasing wavelet frequency. When the incidence angle is between $40^{\circ}$ and $45^{\circ}$, the reflection coefficients calculated for different wavelet frequencies are approximately equal. For the converted $S$ wave, the reflection coefficient increases with increasing wavelet frequency.

Finally, we investigate the effect of the coalbed thickness on the reflection coefficient. The thickness is set from 3 to $11 \mathrm{~m}$ at $2-\mathrm{m}$ intervals, and the results are shown in Fig. 12.

The changes in the wave reflection coefficient curves seen in Fig. 12 are similar to those seen in Fig. 11. The reflection coefficient increases with increasing coalbed thickness. When the incidence angle is between $40^{\circ}$ and $45^{\circ}$, the $P$-wave reflection coefficients calculated for different coalbed thicknesses are approximately equal.

After discussing the changes in the reflection coefficient for an azimuthal angle of $0^{\circ}$, we consider the simulation of the wave propagation at an azimuthal angle of $90^{\circ}$. Figure 13 shows the effect of the crack density, crack aspect ratio, crack filler, wavelet frequency, and thickness of the coalbed on the $P$-wave reflection coefficient at the azimuth of $90^{\circ}$ (parallel to the cracks system).

In Figure 13a-c, there is no change in the $P$-wave reflection coefficient. Hence, a crack does not affect the reflection coefficient when the azimuthal angle is parallel to the cracks. In Figure 13d-e, the $P$-wave reflection coefficient increases with increasing wavelet frequency and coalbed thickness. As the incidence angle tends to $45^{\circ}$, the reflection coefficients become approximately equal. Therefore, when wave propagation is simulated at an azimuthal angle of $90^{\circ}$, only background isotropic parameters of the coalbed affect the reflection coefficient. This result is similar to that of Zhang et al. (1997) computed using a three-layer isotropic model. 
(a) Crack density

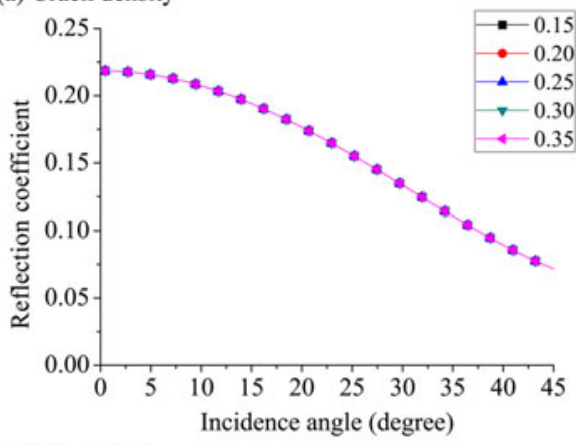

(c) Crack filler

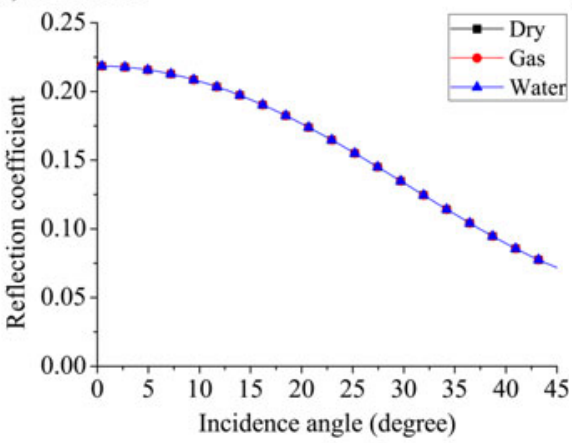

(e) Thickness of the coalbed

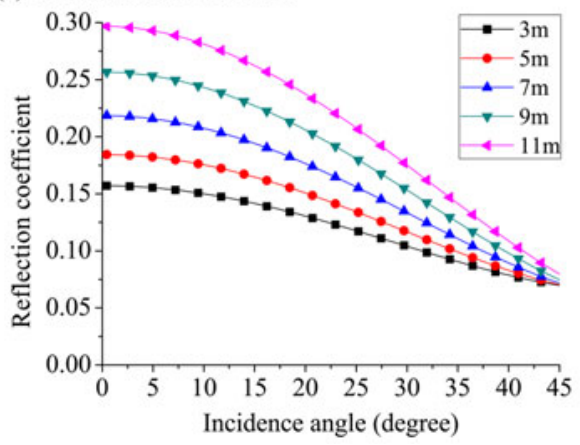

(b) Crack aspect radio

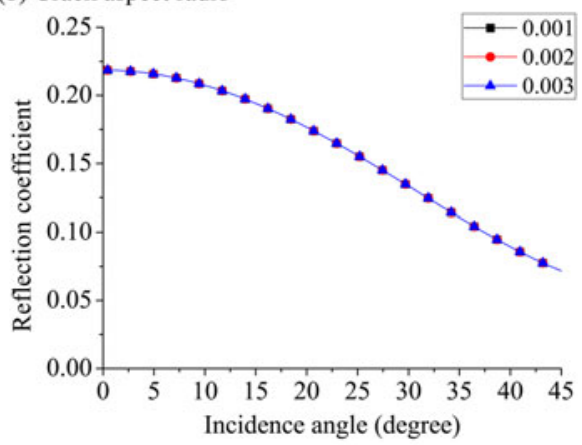

(d) Wavelet frequency

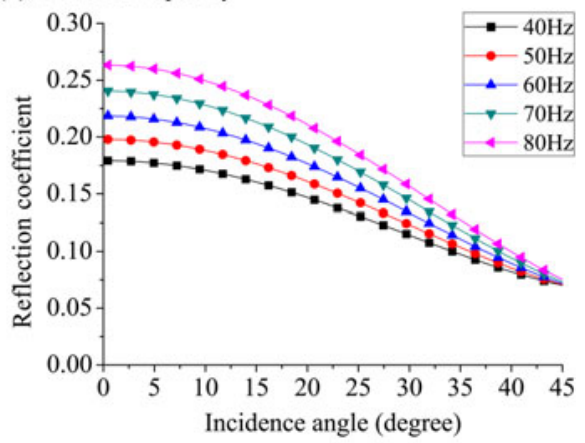

Fig. 13. Effects of coalbed parameters on the $P$-wave reflection coefficient (azimuth of $90^{\circ}$ ).

To investigate the seismic amplitude variation with the offset, we simulated a two-dimensional survey line at a 5-m receiver spacing at the surface. The $P$-wave source of the Ricker wavelet with a dominant frequency of $60 \mathrm{~Hz}$ is located at the starting point of the survey line. The time sampling interval is $0.5 \mathrm{~ms}$. We use three-layer model for an azimuthal angle of $0^{\circ}$ and a gas-saturated crack and the same other parameters as in the previous example. Figure 14 shows the synthetic common shot gather for the $P$ and 

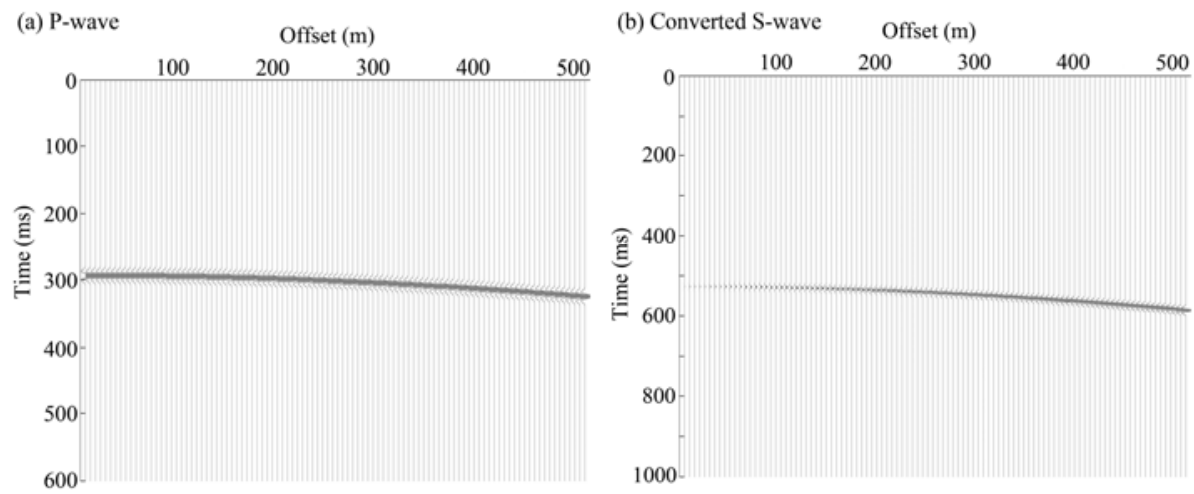

Fig. 14. Common shot gather.

converted $P S$ waves. The offset varies from 0 to $500 \mathrm{~m}$ at intervals of $5 \mathrm{~m}$. The synthetic seismogram shows the same general trend in the AVO response as observed in the previous example.

The seismic amplitude obtained by forward modeling has been used to interpret variations in physical parameters of the fracture and coalbed. In practice, by comparing real seismic records from a coalfield with the synthetic seismogram, we can inverse some parameters of the coalbed according to the amplitude. This has important significance in detecting CBM.

\section{CONCLUSION}

In this paper, we built a three-layer model to describe AVO responses in an anisotropic coalbed. The top and bottom layers were isotropic, while the middle layer was a fractured coalbed considered as an EDA medium. Using the model, we simulated wave propagation and discussed individual reflections from the top and bottom of the coalbed. Employing Cheng's theory, Kelvin-Christoffel equations and boundary conditions, an exact formula to calculate the reflection coefficient was derived. We modelled reflection coefficients of the $P$ and converted $P S$ waves for the azimuths $0^{\circ}$ and $90^{\circ}$. We demonstrated that the reflection coefficient of the $P$ wave increases while the converted $P S$ wave decreases with the increasing incidence angle.

The crack density, crack aspect ratio, crack filler, wavelet frequency, and thickness of the coalbed have some effect on the reflection coefficient at an azimuthal angle of $0^{\circ}$. The results show that the intercept and slope of $P$ wave reflection coefficient curves increase with increasing crack density, wavelet frequency, and coalbed thickness. In a certain range of the incidence angle, $P$-wave reflection coefficients are approximately equal. For the converted $S$ wave, there is also an increasing tendency for the reflection coefficient. Additionally, the reflection coefficients are considerably affected by 
gas-saturated and water-saturated cracks. The crack aspect ratio has little effect on the reflection coefficient. When the azimuthal angle of fracture is parallel to the cracks, the differences in fracture parameters do not affect the reflected AVO signature, and the coalbed can be considered an isotropic medium.

To analyze the variation in seismic amplitude, we created a synthetic seismogram by forward calculation. The information of the amplitude was used to detect changes of cracks in the coalbed. In particular, AVO curves were notably different for gas-saturated cracks and water-saturated cracks in a coalbed. This finding can be applied to CBM detection. Although there will be some possible obstacles like data quality, effect from the stress, temperature, etc., our study shows that in the presence of the methane filled coal fractures we can expect changes in the amplitudes which can be possibly in the future inverted for the density of the fractures and may be for the methane content.

Acknowledgments. We greatly appreciate the support of the National 973 Program of China (2009CB219603, 2010CB226800, 2009CB724601), National Natural Science Foundation of China (Major Program) (50490271, 40672104), National Natural Science Foundation of China (General Program) (40874071), Joint Fund of Coal (U1261203), the National Science and Technology Pillar Program in the Eleventh Five-year Plan Period (2012BAB13B01, 2012BAC10B03), and the China Geological Survey Project (1212011220798, 1212011220188).

\section{Appendices}

\section{A. Elastic wave equation and Kelvin-Christoffel equation for anisotropic media}

As the theoretical basis of wave mechanics, the elastic wave equation has been widely used to describe seismic wave propagation in isotropic and anisotropic media. In anisotropic media, the strain produced by a stress of any kind is proportional to the stress. Hook's law explains the stress-strain relationship and can be written using matrix notation as (Carcione 2007):

$$
\boldsymbol{\sigma}=\mathbf{C} \cdot \mathbf{e},
$$

where $\boldsymbol{\sigma}$ is the stress tensor, $\mathbf{e}$ is the strain tensor, and $\mathbf{C}$ is the stiffness tensor of $6 \times 6$ matrices. From Newton's second law, which describes the dynamic 
process among the stress, strain, and displacement, we get the general equation of motion in the absence of body forces

$$
\rho \frac{\partial^{2}}{\partial t^{2}} \mathbf{u}=\nabla \cdot \boldsymbol{\sigma},
$$

where $\rho$ is the density, $\mathbf{u}$ is the displacement vector, and $\nabla$ using the matrix representation is given by (Auld 1990)

$$
\nabla=\left[\begin{array}{lllllr}
\partial_{x} & 0 & 0 & 0 & \partial_{z} & \partial_{y} \\
0 & \partial_{y} & 0 & \partial_{z} & 0 & \partial_{x} \\
0 & 0 & \partial_{z} & \partial_{y} & \partial_{x} & 0
\end{array}\right]
$$

Equation A2 can be written in another form according to the relationship among the stress, strain and displacement

$$
\rho \frac{\partial^{2}}{\partial t^{2}} \mathbf{u}=\nabla \cdot \mathrm{C} \cdot \nabla^{\mathrm{T}} \cdot \mathbf{u} .
$$

Equation A4 is the anisotropic elastic wave equation.

A general wave solution for the displacement vector is written as (Ali and Jakobsen 2011)

$$
\mathbf{u}=\mathbf{P} A_{0} e^{i(\mathbf{k} \cdot \mathbf{x}-\omega t)},
$$

where

$$
\mathbf{k}=k\left(l_{x}, l_{y}, l_{z}\right) .
$$

Here $\mathbf{u}=\left(u_{x}, u_{y}, u_{z}\right)^{T}$ is the displacement vector, $\mathbf{P}=\left(p_{x}, p_{y}, p_{z}\right)^{T}$ is the polarization vector, $A_{0}$ is the wave amplitude, $\mathbf{k}$ is the wave-number vector and $l_{x}, l_{y}$, and $l_{z}$ are the direction cosines. The spatial differential operator in Eq. A3 can be replaced by

$$
\nabla \rightarrow i k\left[\begin{array}{cccccc}
l_{x} & 0 & 0 & 0 & l_{z} & l_{y} \\
0 & l_{y} & 0 & l_{z} & 0 & l_{x} \\
0 & 0 & l_{z} & l_{y} & l_{x} & 0
\end{array}\right]=i k \mathbf{L} .
$$

Substitution of Eqs. A5-A7 into Eq. A4 gives the Kelvin-Christoffel equation (Musgrave 1970)

$$
\left[\begin{array}{ccc}
\Gamma_{11}-\rho v^{2} & \Gamma_{12} & \Gamma_{13} \\
\Gamma_{21} & \Gamma_{22}-\rho v^{2} & \Gamma_{23} \\
\Gamma_{31} & \Gamma_{32} & \Gamma_{33}-\rho v^{2}
\end{array}\right]\left[\begin{array}{c}
p_{x} \\
p_{y} \\
p_{z}
\end{array}\right]=0,
$$


(a) Dry fractures

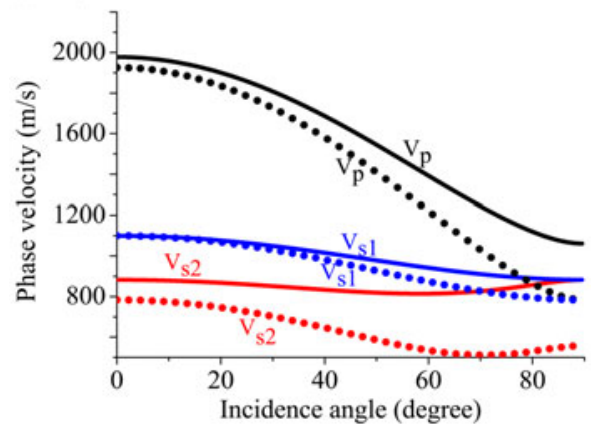

(c) Water-saturated fractures

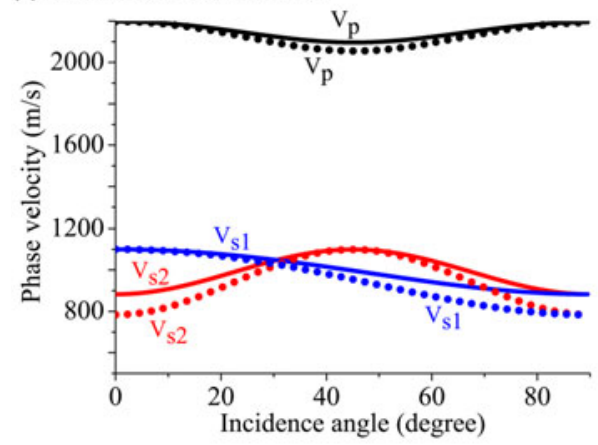

(b) Gas-saturated fractures

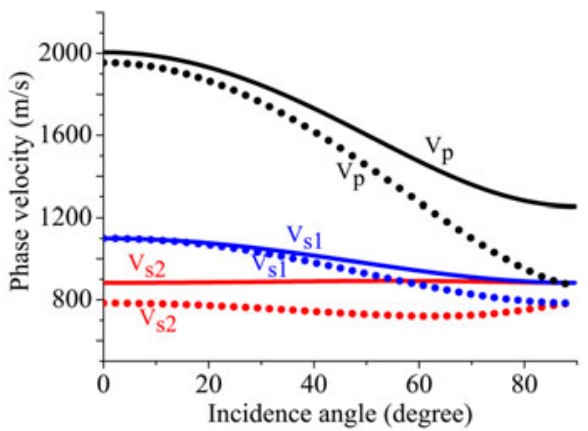

Fig. A1. Variations in the phase velocities of the three waves for angle of incidence between $0^{\circ}$ and $90^{\circ}$ with crack density $\varepsilon=0.2$ (solid line) and $\varepsilon=0.3$ (dashed line).

where

$$
\boldsymbol{\Gamma}=\left[\begin{array}{lll}
\Gamma_{11} & \Gamma_{12} & \Gamma_{13} \\
\Gamma_{21} & \Gamma_{22} & \Gamma_{23} \\
\Gamma_{31} & \Gamma_{32} & \Gamma_{33}
\end{array}\right]=\mathbf{L} \cdot \mathbf{C} \cdot \mathbf{L}^{T}
$$

is the Christoffel matrix. Because the polarization vector is nonzero, Eq. A8 has a nonzero solution, i.e.,

$$
\operatorname{det}\left(\boldsymbol{\Gamma}-\rho v^{2} \mathbf{I}_{3}\right)=0 .
$$

After solving Eqs. 9 and 10, we can obtain the wave velocity $v$ and polarization vector $\mathbf{P}$. Figure A1 shows the vertical sections of the $P$-, $S V$-, and $S H$-wave phase velocities for gas-saturated, water-saturated, and dry cracks. The background parameters are the same as in Fig. 2 except that the crack density is taken as 0.2 and 0.3 . From Fig. A1, it is obvious that the crack filler strongly affects the velocities of $P, S V$, and $S H$ waves. Additionally, the three wave velocities reduce as the crack density increases. 


\section{B. Reflection of a plane wave in a two-layer anisotropic model}

The two-layer model is shown in Fig. 3a. Two homogeneous anisotropic half-spaces (EDA1 and EDA2) are separated by a horizontal interface. A plane $P$-wave incident on the interface generates three plane waves with mutually orthogonal polarization directions: $P, S V$, and $S H$ waves. When the incident $P$-wave travels in the $[x, z]$-plane, we need to consider the coupling of $P$ and $S V$ waves at the interface. However, the third wave type, the $S H$ wave, is polarized perpendicular to the $[x, z]$-plane and is not excited by incident $P$ and $S V$ waves. Additionally, it is decoupled from the $P$ and $S V$ waves. Thus, we only need to solve the transmission and reflection coefficients of the $P$ and $S V$ waves due to the continuity requirements at the plane horizontal interface in the $[x, z]$-plane (azimuth of $0^{\circ}$ ). The wave propagation in the $[y, z]$-plane (azimuth of $90^{\circ}$ ) can be described in the same way. Figure B1 shows the plane wave propagation in the two-layer model for an azimuthal angle of $0^{\circ}$. The plane $P$-wave $P$ incident at the interface generates four waves: reflected $P P_{1}$ and $P S_{1}$ waves, and transmitted $P P_{2}$ and $P S_{2}$ waves.

Next, we begin to derive the exact solution for reflection and transmission coefficients as a function of the incidence angle. Boundary conditions have to be applied to solve for the reflection and transmission coefficients. We assume that the stiffness tensors of $\mathrm{EDA}^{(1)}$ and $\mathrm{EDA}^{(2)}$ are $\mathbf{C}^{(\mathbf{1})}$ and $\mathbf{C}^{(\mathbf{2})}$ for an azimuthal angle of $0^{\circ}$. Using Cheng's theory (1993) mentioned above, elastic stiffness tensors can be computed from the known background velocity, crack density, crack aspect ratio, and crack filler. The stress-strain relationship, explained by Hooke's law in Appendix A, can then be written as

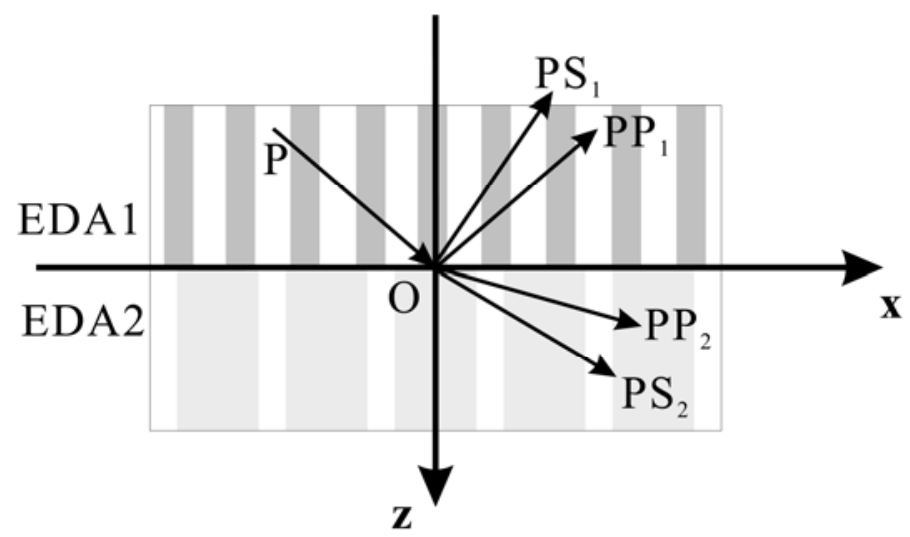

Fig. B1. Wave propagation in the two-layer model. 


$$
\left[\begin{array}{l}
\sigma_{x x}^{(j)} \\
\sigma_{y y}^{(j)} \\
\sigma_{z z}^{(j)} \\
\sigma_{y z}^{(j)} \\
\sigma_{x z}^{(j)} \\
\sigma_{x y}^{(j)}
\end{array}\right]=\left[\begin{array}{cccccc}
c_{11}^{(j)} & c_{13}^{(j)} & c_{13}^{(j)} & 0 & 0 & 0 \\
c_{13}^{(j)} & c_{22}^{(j)} & c_{23}^{(j)} & 0 & 0 & 0 \\
c_{13}^{(j)} & c_{23}^{(j)} & c_{33}^{(j)} & 0 & 0 & 0 \\
0 & 0 & 0 & c_{44}^{(j)} & 0 & 0 \\
0 & 0 & 0 & 0 & c_{55}^{(j)} & 0 \\
0 & 0 & 0 & 0 & 0 & c_{55}^{(j)}
\end{array}\right]\left[\begin{array}{c}
e_{x x}^{(j)} \\
e_{y y}^{(j)} \\
e_{z z}^{(j)} \\
e_{y z}^{(j)} \\
e_{x z}^{(j)} \\
e_{x y}^{(j)}
\end{array}\right], \quad(j=1,2),
$$

where $\mathrm{C}^{(j)}=\left[c_{m n}^{(j)}\right](m, n=1,2, \ldots, 6)$ is the stiffness tensor of $\operatorname{EDA}^{(j)}$, $\boldsymbol{\sigma}^{(j)}=\left[\sigma_{x x}^{(j)}, \sigma_{y y}^{(j)}, \sigma_{z z}^{(j)}, \sigma_{y z}^{(j)}, \sigma_{x z}^{(j)}, \sigma_{x y}^{(j)}\right]^{T}$ is the stress tensor of $\mathrm{EDA}^{(j)}$, and $\mathbf{e}^{(j)}=\left[e_{x x}^{(j)}, e_{y y}^{(j)}, e_{z z}^{(j)}, e_{y z}^{(j)}, e_{x z}^{(j)}, e_{x y}^{(j)}\right]^{T}$ is the strain tensor of $\mathrm{EDA}^{(j)}$.

Meanwhile, the strain-displacement relationship can be expressed as

$$
\begin{gathered}
e_{x x}^{(j)}=\frac{\partial u_{x}^{(j)}}{\partial x}, \quad e_{y y}^{(j)}=\frac{\partial u_{y}^{(j)}}{\partial y}, \quad e_{z z}^{(j)}=\frac{\partial u_{z}^{(j)}}{\partial z}, \\
e_{y z}^{(j)}=\frac{\partial u_{z}^{(j)}}{\partial y}+\frac{\partial u_{y}^{(j)}}{\partial z}, \quad e_{x z}^{(j)}=\frac{\partial u_{z}^{(j)}}{\partial x}+\frac{\partial u_{x}^{(j)}}{\partial z}, \quad e_{x y}^{(j)}=\frac{\partial u_{x}^{(j)}}{\partial y}+\frac{\partial u_{y}^{(j)}}{\partial x} \quad(j=1,2),
\end{gathered}
$$

where $u_{x}^{(j)}, u_{y}^{(j)}$, and $u_{z}^{(j)}$ are components of the displacement vector $\mathbf{u}^{(j)}$ in the directions of $x, y$, and $z$. The continuity of stress at the reflecting horizon yields (assuming that the depth of the interface is zero; i.e., $z=0$ )

$$
\sigma_{z z}^{(1)}=\sigma_{z z}^{(2)}, \quad \sigma_{z x}^{(1)}=\sigma_{z x}^{(2)} .
$$

Substituting Eq. B2 into B1, we have

$$
\sigma_{z z}^{(j)}=c_{13}^{(j)} \frac{\partial u_{x}^{(j)}}{\partial x}+c_{33}^{(j)} \frac{\partial u_{z}^{(j)}}{\partial z}, \quad \sigma_{x z}^{(j)}=c_{66}^{(j)}\left(\frac{\partial u_{z}^{(j)}}{\partial x}+\frac{\partial u_{x}^{(j)}}{\partial z}\right) .
$$

Substituting Eq. (B4) into (B3), we have

$$
\begin{gathered}
c_{13}^{(1)} \frac{\partial u_{x}^{(1)}}{\partial x}+c_{33}^{(1)} \frac{\partial u_{z}^{(1)}}{\partial z}=c_{13}^{(2)} \frac{\partial u_{x}^{(2)}}{\partial x}+c_{33}^{(2)} \frac{\partial u_{z}^{(2)}}{\partial z}, \\
c_{66}^{(1)}\left(\frac{\partial u_{z}^{(1)}}{\partial x}+\frac{\partial u_{x}^{(1)}}{\partial z}\right)=c_{66}^{(2)}\left(\frac{\partial u_{z}^{(2)}}{\partial x}+\frac{\partial u_{x}^{(2)}}{\partial z}\right) .
\end{gathered}
$$

Equation A5 is a function of the displacement vector of a plane wave. In the $[x, z]$-plane, the displacement vector $\mathbf{u}$ has the form

$$
\mathbf{u}_{k}=\left(f_{k}, h_{k}\right) A_{k} \exp \left[i \omega\left(p_{k} x+r_{k} z-t\right)\right], \quad k=1,2, \ldots, 5,
$$


where $\mathbf{P}_{k}=\left(f_{k}, h_{k}\right)$ is the unit polarization vector, $A_{k}$ is the wave amplitude, $p_{k}$ is horizontal slowness with respect to the $x$-axis, and $r_{k}$ is vertical slowness with respect to the $z$-axis. $k=1,2, \ldots, 5$ denote the incident $P$-wave $P$, reflected $P$-wave $P P_{1}$, reflected converted $S$-wave $P S_{1}$, transmitted $P$-wave $P P_{2}$, and transmitted converted $S$-wave $P S_{2}$, respectively.

At the top of the interface, the total displacement of waves propagating in $\operatorname{EDA}^{(1)}$ is written as

$$
\begin{aligned}
& u_{x}^{(1)}=\sum_{k=1}^{3} f_{k} A_{k} \exp \left[i \omega\left(p_{k} x+r_{k} z-t\right)\right], \\
& u_{z}^{(1)}=\sum_{k=1}^{3} h_{k} A_{k} \exp \left[i \omega\left(p_{k} x+r_{k} z-t\right)\right] .
\end{aligned}
$$

Similarly, the total displacement at the bottom of the interface is written as

$$
\begin{aligned}
& u_{x}^{(2)}=\sum_{k=4}^{2} f_{k} A_{k} \exp \left[i \omega\left(p_{k} x+r_{k} z-t\right)\right], \\
& u_{z}^{(2)}=\sum_{k=4}^{2} h_{k} A_{k} \exp \left[i \omega\left(p_{k} x+r_{k} z-t\right)\right] .
\end{aligned}
$$

From the boundary condition, which describes the continuity of displacement, we obtain

$$
\begin{aligned}
\sum_{k=1}^{3} f_{k} A_{k} \exp \left[i \omega\left(p_{k} x-t\right)\right] & =\sum_{k=4}^{2} f_{k} A_{k} \exp \left[i \omega\left(p_{k} x-t\right)\right], \\
\sum_{k=1}^{3} h_{k} A_{k} \exp \left[i \omega\left(p_{k} x+r_{k} z-t\right)\right] & =\sum_{k=4}^{2} h_{k} A_{k} \exp \left[i \omega\left(p_{k} x+r_{k} z-t\right)\right] .
\end{aligned}
$$

From the solution of the Kelvin-Christoffel equation and Snell's law, we conclude that the horizontal slowness shown in Fig. B1 is the same for all waves. Letting $p_{k}=p$, Eqs. $\mathrm{B} 9$ and $\mathrm{B} 10$ can be reduced to

$$
\sum_{k=1}^{3} f_{k} A_{k}=\sum_{k=4}^{2} f_{k} A_{k}, \quad \sum_{k=1}^{3} h_{k} A_{k}=\sum_{k=4}^{2} h_{k} A_{k} .
$$

Solving Eqs. B5 and B11, we have

$$
\mathrm{M}\left(A_{2}, A_{3}, A_{4}, A_{5}\right)^{T}=\mathrm{N}\left(A_{1}\right),
$$

where $\mathrm{M}$ and $\mathrm{N}$ are $4 \times 4$ matrices that contain the elements of the stiffness matrix, polarization and slownesses. It is well known that the reflection and transmission coefficients are defined as

$$
R_{p p}=\frac{A_{2}}{A_{1}}, \quad R_{p s}=\frac{A_{3}}{A_{1}}, \quad T_{p p}=\frac{A_{4}}{A_{1}}, \quad T_{p s}=\frac{A_{5}}{A_{1}} .
$$

Equation B12 can then be written as 


$$
\left(R_{p p}, R_{p s}, T_{p p}, T_{p s}\right)=\mathrm{M}^{-1} \mathrm{~N} .
$$

Equation B14 represents the exact solution for reflection and transmission coefficients at an interface of EDA media.

\section{References}

Aki, K., and P.G. Richards (1980), Quantitative Seismology, W.H. Freeman \& Co, New York.

Ali, A., and M. Jakobsen (2011), Seismic characterization of reservoirs with multiple fracture sets using velocity and attenuation anisotropy data, J. Appl. Geophys. 75, 3, 590-602, DOI: 10.1016/j.jappgeo.2011.09.003.

Auld, B.A. (1990), Acoustic Fields and Waves in Solids, Krieger Publ. Malabar.

Bachu, S., and S. Bell (2001), Stress regime in the Cretaceous succession of the Alberta basin: A predictor for coal bed methane producibility. In: Rock the Foundation Convention, 18-22 June 2001, Canadian Society of Petroleum Geologists, Calgary, Canada, 003-1-003-5.

Bakulin, A., V. Grechka, and I. Tsvankin (2000a), Estimation of fracture parameters from reflection seismic data - Part I: HTI model due to a single fracture set, Geophysics 65, 6, 1788-1802, DOI: 10.1190/1.1444863.

Bakulin, A., V. Grechka, and I. Tsvankin (2000b), Estimation of fracture parameters from reflection seismic data - Part II: Fractured models with orthorhombic symmetry, Geophysics 65, 6, 1803-1817, DOI: 10.1190/1.1444864.

Bortfeld, R. (1961), Approximations to the reflection and transmission coefficients of plane longitudinal and transverse waves, Geophys. Prospect. 9, 4, 485502, DOI: $10.1111 /$ j.1365-2478.1961.tb01670.x.

Carcione, J.M. (2007), Wave Fields in Real Media: Wave Propagation in Anisotropic, Anelastic, Porous and Electromagnetic Media, Elsevier, Amsterdam.

Castagna, J.P., H.W. Swan, and D.J. Foster (1998), Framework for AVO gradient and intercept interpretation, Geophysics 63, 3, 948-956, DOI: 10.1190/ 1.1444406.

Chen, W. (1995), AVO in azimuthally anisotropic media fracture detection using P-wave data and a seismic study of naturally fractured tight gas reservoirs, Department of Geophysics, School of Earth Sciences, Stanford University, USA.

Cheng, C.H. (1993), Crack models for a transversely isotropic medium, J. Geophys. Res. 98, B1, 675-684, DOI: 10.1029/92JB02118.

Crampin, S. (1984), Effective anisotropic elastic constants for wave propagation through cracked solids, Geophys. J. Int. 76, 1, 135-145, DOI: 10.1111/ j.1365-246X.1984.tb05029.x. 
Crampin, S. (1989), Suggestions for a consistent terminology for seismic anisotropy, Geophys. Prospect. 37, 7, 753-770, DOI: 10.1111/j.1365-2478.1989. tb02232.x.

Du, S.T. (1996), Seismic Wave Dynamics, China University of Petroleum Press, Dongying, China (in Chinese).

Eshelby, J.D. (1957), The determination of the elastic field of an ellipsoidal inclusion, and related problems, Proc. Roy. Soc. London A 241, 1226, 376-396, DOI: $10.1098 /$ rspa.1957.0133.

Gochioco, L.M. (1991), Tuning effect and interference reflections from thin beds and coal seams, Geophysics 56, 8, 1288-1295, DOI: 10.1190/1.1443151.

$\mathrm{Hu}$, Y.R., and G.A. McMechan (2007), Imaging mining hazards within coalbeds using prestack wave equation migration of in-seam seismic survey data: A feasibility study with synthetic data, J. Appl. Geophys. 63, 1, 24-34, DOI: 10.1016/j.jappgeo.2007.03.002.

Hudson, J.A. (1980), Overall properties of a cracked solid, Math. Proc. Cambridge Philos. Soc. 88, 2, 371-384, DOI: 10.1017/S0305004100057674.

Hudson, J.A. (1981), Wave speeds and attenuation of elastic waves in materials containing cracks, Geophys. J. Int. 64, 1, 133-150, DOI: 10.1111/j.1365246X.1981.tb02662.x.

Komatitsch, D., and J. Tromp (2003), A perfectly matched layer absorbing boundary condition for the second-order seismic wave equation, Geophys. J. Int. 154, 1, 146-153, DOI: 10.1046/j.1365-246X.2003.01950.x.

Meissner, R., and E. Meixner (1969), Deformation of seismic wavelets by thin layers and layered boundaries, Geophys. Prospect. 17, 1, 1-27, DOI: 10.1111/ j.1365-2478.1969.tb02069.x.

Musgrave, M.J.P. (1970), Crystal Acoustics: Introduction to the Study of Elastic Waves and Vibrations in Crystals, Holden-Day, San Francisco.

Ostrander, W.J. (1984), Plane-wave reflection coefficients for gas sands at nonnormal angles of incidence, Geophysics 49, 10, 1637-1648, DOI: 10.1190/ 1.1441571 .

Peng, S.P., H.J. Chen, R.Z. Yang, Y.F. Gao, and X.P. Chen (2006), Factors facilitating or limiting the use of AVO for coal-bed methane, Geophysics 71, 4, C49-C56, DOI: 10.1190/1.2217137.

Ramos, A.C.B., and T.L. Davis (1997), 3-D AVO analysis and modeling applied to fracture detection in coalbed methane reservoirs, Geophysics 62, 6, 16831695, DOI: 10.1190/1.1444268.

Rüger, A. (1997), P-wave reflection coefficients for transversely isotropic models with vertical and horizontal axis of symmetry, Geophysics 62, 3, 713-722, DOI: $10.1190 / 1.1444181$.

Rutherford, S.R., and R.H. Williams (1989), Amplitude-versus-offset variations in gas sands, Geophysics 54, 6, 680-688, DOI: 10.1190/1.1442696. 
Shuck, E.L., T.L. Davis, and R.D. Benson (1996), Multicomponent 3-D characterization of a coalbed methane reservoir, Geophysics 61, 2, 315-330, DOI: 10.1190/1.1443961.

Shuey, R.T. (1985), A simplification of the Zoeppritz equations, Geophysics 50, 4, 609-614, DOI: 10.1190/1.1441936.

Wright, J. (1987), The effects of transverse isotropy on reflection amplitude versus offset, Geophysics 52, 4, 564-567, DOI: 10.1190/1.1442325.

Zhang, A.M., Y. Wang, and S.Z. Zhao (1997), Study on the AVO model and AVO character of coal seams with different thickness, J. China Univ. Min. Technol. 26, 3, 36-41 (in Chinese).

Zoeppritz, K. (1919), Erdbebenwellen VII B. On the reflection and penetration of seismic waves through unstable layers, Nachr. Gesellsch. Wissensch. Göttingen, Math.-Physik. Kl. 1919, 66-84.

Received 11 December 2013

Received in revised form 7 May 2014

Accepted 24 June 2014 